\title{
Narrow-band detection of propagating coherent acoustic phonons in piezoelectric InGaN/GaN multiple-quantum wells
}

\author{
Cheng-Ying Chen, Yu-Chieh Wen, Hung-Ping Chen, and Tzu-Ming Liu \\ Department of Electrical Engineering and Graduate Institute of Photonics and Optoelectronics, \\ National Taiwan University, Taipei 10617, Taiwan \\ Chang-Chi Pan and Jen-Inn Chyi \\ Optical Sciences Center, National Central University, Chung-Li 32054, Taiwan \\ Chi-Kuang Sun ${ }^{\text {a) }}$ \\ Department of Electrical Engineering and Graduate Institute of Photonics and Optoelectronics, \\ National Taiwan University, Taipei 10617, Taiwan and Research Center for Applied Sciences, Academia \\ Sinica, Taipei 10617, Taiwan
}

(Received 12 June 2007; accepted 27 August 2007; published online 24 September 2007)

\begin{abstract}
The authors demonstrated that the piezoelectric superlattice, can serve as narrow-band detectors for propagating coherent longitudinal acoustic phonons at multiple frequencies corresponding to the spatial frequency of the superlattice and its higher harmonics, and its detection bandwidth is determined by the total structure width. By optically exciting a broadband propagating longitudinal acoustic pulse from a thin $\mathrm{Ni}$ film, the authors studied the acoustic spectral sensitivity function of a ten-period $\operatorname{In}_{0.12} \mathrm{Ga}_{0.88} \mathrm{~N} / \mathrm{GaN}$ multiple quantum well. Because the barriers $(19 \mathrm{~nm})$ and wells $(3.6 \mathrm{~nm})$ are of different widths, the second detection band, corresponding to the second harmonic of the fundamental frequency, can be resolved. (C) 2007 American Institute of Physics.
\end{abstract}

[DOI: $10.1063 / 1.2785126]$

It has been shown that ultrafast optical excitation of GaAs/AlAs and $\mathrm{GaAs} / \mathrm{Al}_{x} \mathrm{Ga}_{1-x} \mathrm{As}$ superlattice structures ${ }^{1,2}$ and piezoelectric $\mathrm{InGaN} / \mathrm{GaN}$ multiple quantum wells ${ }^{3,4}$ (MQWs) results in the generation of coherent acoustic phonons (CAPs). However, in these previous experiments the semiconductor structures were not only a generator but also a detector of the CAPs, and few experiments were conducted where the generation and the detection of CAPs were spatially separated. ${ }^{5,6}$ For piezoelectric superlattices, its spectral sensitivity function for coherent acoustic phonon detection has never been independently measured. ${ }^{7}$ This motivated us to generate broadband acoustic phonons in a separated layer ${ }^{8,9}$ to investigate the detection sensitivity of a periodic piezoelectric multilayer. In this paper, we demonstrate that the piezoelectric superlattice with a low impedence mismatch between adjacent layers can serve as narrow-band CAP detectors at multiple frequencies corresponding to the spatial frequency of the MQWs and its higher harmonics. We adopt $\operatorname{In}_{0.12} \mathrm{Ga}_{0.88} \mathrm{~N} / \mathrm{GaN} \mathrm{MQW}$ as our studied narrow-band CAP detectors. With a broad-spectrum propagating longitudinal acoustic pulse (LAP), which resulted from an $\sim 3-6 \mathrm{~nm}$ Ni film after optical impulse excitation, we studied the acoustic spectral sensitivity function for optical transmission of the ten-period $\operatorname{In}_{0.12} \mathrm{Ga}_{0.88} \mathrm{~N} / \mathrm{GaN}$ MQW. With an asymmetric well/barrier ratio and a broad enough LAP bandwidth, the InGaN/GaN MQW experimentally exhibited two narrow detection bands of which the central frequencies correspond to the period of the MQW and its second harmonic, respectively, ${ }^{10}$ and the CAP detection bandwith is determined by the total MQW width.

\footnotetext{
a) Author to whom correspondence should be addressed. Tel. +886-2-33665085. FAX: +886-2-33663614. Electronic mail: sun@cc.ee.ntu.edu.tw
}

In periodic semiconductor multilayer structures, due to the zone folding of the phonon branches into mini-Brillouin zones with a dimension of $\pi / \Lambda$ and the impedance mismatch between layers, the phonon stop bands appear at acoustic wave vectors of $q=n \pi / \Lambda$, where $\Lambda$ is one-period width of the multilayer structure and $n$ is an integer. The acoustic phonons with a stop band frequency cannot propagate in these structures and will be reflected due to the Bragg condition. ${ }^{11}$ In previous works, phonon filters based on stop bands in periodic semiconductor multilayers have been realized. ${ }^{12-15}$ As a result, when the propagating LAP incidents upon the periodic semiconductor multilayers with a large acoustic impedance mismatch, it will be hard for this structure to detect propagating phonons with a stop band frequency. In contrast to the previous stop band filter result, ${ }^{12-15}$ with a low impedence mismatch between piezoelectric multilayers with a limited number of layers, for example, the ten-period $\operatorname{In}_{0.12} \mathrm{Ga}_{0.88} \mathrm{~N} / \mathrm{GaN}$ MQW, ${ }^{16,17}$ we expect that the piezoelectric multilayer structure will not only allow transmission of the acoustic phonons with an acoustic wave vector of $q=2 n \pi / \Lambda$ with negligible interfacial reflections but can also provide narrow-band CAP detection through the strain induced modulation in the quantum confined potentials, ${ }^{4,718}$ with the central frequency of detection corresponding to $q=2 n \pi / \Lambda$ and its higher harmonics.

The sample we used in this experiment was grown on an $\sim 3.4$ - $\mu \mathrm{m}$-thick wurtzite GaN layer on a $c$-axis sapphire substrate by metal organic chemical vapor deposition. It has ten periods of $c$-axis orientated $\operatorname{In}_{0.12} \mathrm{Ga}_{0.88} \mathrm{~N} / \mathrm{GaN}(3.6 / 19 \mathrm{~nm})$ layers as the narrow-band CAP detector, and the structure was confirmed by transmission electron microscopy, as shown in Fig. 1. The room temperature photoluminescence of the MQW is with a peak at $\sim 460 \mathrm{~nm}$. On top of the $\mathrm{MQW}$, the sample was capped with a $17 \mathrm{~nm}$ layer of unintentionally doped GaN. Finally, we deposited an $\sim 3-6 \mathrm{~nm}$ 


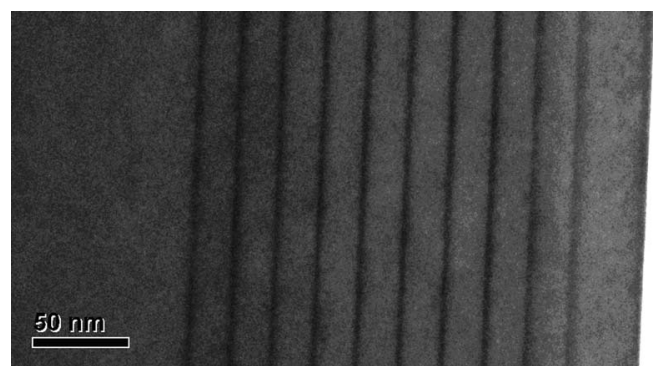

FIG. 1. Transmission electron micrograph of the ten-period $\mathrm{In}_{0.12} \mathrm{Ga}_{0.88} \mathrm{~N} / \mathrm{GaN} \mathrm{MQW}$. The first layer on right hand side includes the 17-nm-thick undoped GaN cap layer of and one $\sim 19$-nm-thick barrier layer. The left hand side shows one part of the $\sim 3.4 \mu \mathrm{m} \mathrm{GaN} \mathrm{layer.} \mathrm{The}$ quantum well width is $\sim 3.6 \mathrm{~nm}$ and the barrier width is $\sim 19 \mathrm{~nm}$.

Ni film on top of the cap layer as a generator of LAP, and the uncertainty of the Ni film thickness came from limited deposition thickness control of the thermal coater. Within the thickness uncertainty, the $\mathrm{Ni}$ film can generate broadspectrum acoustic phonons at least up to $800 \mathrm{GHz}$, enough for our current study. Because the acoustic impedance of $\mathrm{Ni}$ is similar to that of $\mathrm{GaN}\left(\mathrm{Z}_{\mathrm{Ni}} / \mathrm{Z}_{\mathrm{GaN}} \sim 1.09\right),{ }^{16,17,19}$ the acoustic reflection coefficient between the $\mathrm{Ni}$ film and the undoped GaN cap layer is less than 0.05 .

The experimental setup is a femtosecond transient transmission two-color pump-probe system. ${ }^{20} \mathrm{~A}$ femtosecond Ti: sapphire laser at a $76 \mathrm{MHz}$ repetition rate provided optical pulses at a central wavelength of $800 \mathrm{~nm}$ for optical pumps. Pulses frequency doubled by a Beta-Barium Borate crystal, now at a central wavelength of $400 \mathrm{~nm}$, were used as optical probes. The powers of the pump and the probe beams were adjusted to be 40 and $6.5 \mathrm{~mW}$, respectively. According to previous experimental $^{4,18}$ and theoretical ${ }^{7}$ works on coherent LA phonon oscillations in piezoelectric MQWs, the strain induced modulation in quantum confined energies can be through several mechanisms, including piezoelectric coupling and deformational potential coupling. A detailed theoretical investigation ${ }^{7}$ found that the piezoelectric coupling is the dominating contribution for optical transmission sensitivity function in $\mathrm{In}_{0.1} \mathrm{GaN}$ MQWs. By choosing the probe photon energy above the InGaN quantum well transition but below the bandgap of the $\mathrm{GaN}(\sim 365 \mathrm{~nm})$, similar to previous studies, ${ }^{4}$ we are able to probe the strain induced absorption changes in the quantum well regions. The temporal delays between the pump and the probe pulses were controlled by a translation stage. Finally, the pump and the probe beams were focused onto the sample with spot diameters of $\sim 20$ and $\sim 10 \mu \mathrm{m}$, respectively, and the transmitted probe pulses were detected by a photodetector linked to a lock-in amplifier. After the Ni film was excited by the pump radiation, a propagating LAP was generated and went through the $17 \mathrm{~nm}$ undoped GaN cap layer into the MQW region along the $c$ axis. The absolute amplitude of the strain pulse can be estimated to be on the oder of $3 \times 10^{-4}$, following Ref. 21 . The propagating LAP in the quantum well modulated the piezoelectric field and deformation potential of the InGaN well and caused variation on optical absorption of the probe beam. ${ }^{4,7}$ The transmission changes of the probe beam due to the existence of coherence LA phonons can be conveniently expressed using this equation $\Delta T(t)=\mathfrak{I}^{-1}[F(\omega) S(\omega)]$, where $\mathfrak{I}^{-1}$ denotes the inverse Fourier transform and $F(\omega)$ and $S(\omega)$ are the acoustic spectral sensitivity function for optical transmission of the MQW and the frequency spectrum of the Downloaded 16 Feb 2009 to 140.112.113.225. Redistribution subject

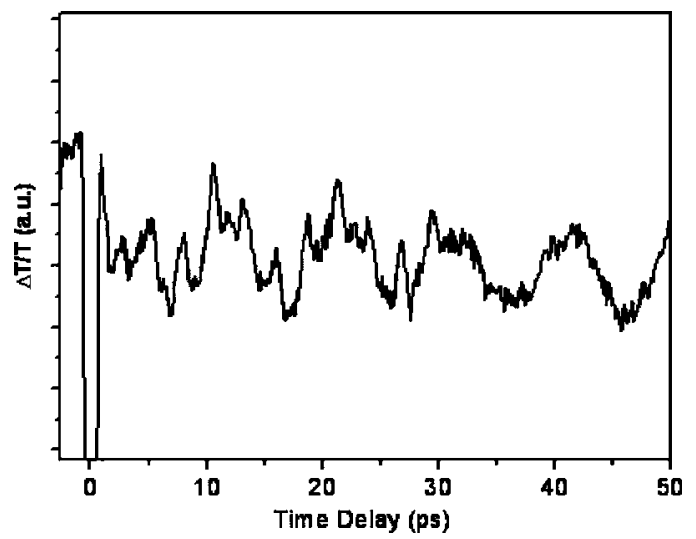

FIG. 2. Measured transient transmission change vs probe time delay.

strain pulse, respectively. The quantity $F(\omega)$ represents the weighted contribution of the strain value to the sample transmission change at a specific acoustic angular frequency $\omega$, and it is related to the wavelength of the probe and the structure of the sample. As shown in Fig. 2, we can see the transmission variation of the probe beam when the LAP propagates into the sample. According to the velocities of the LA modes of $\mathrm{Ni}$ and $\mathrm{GaN}$ along the $c$ axis, ${ }^{16,17,19}$ we can know that the transient transmission changes for the time delay of $\sim 4-30 \mathrm{ps}$ is resulted from the LAP propagating in the $\mathrm{In}_{0.12} \mathrm{Ga}_{0.88} \mathrm{~N} / \mathrm{GaN} \mathrm{MQW}$ region. Hence we use the transient transmission signal between 4 and 30 ps to study the characteristic of the acoustic phonon spectral sensitivity function $F(\omega)$ of the ten-period $\mathrm{In}_{0.12} \mathrm{Ga}_{0.88} \mathrm{~N} / \mathrm{GaN}$ MQW.

In order to investigate its frequency domain response, we performed fast Fourier transformation on the transient transmission signal shown in Fig. 2 for $\sim 4-30 \mathrm{ps,} \mathrm{and} \mathrm{the}$ result is shown in Fig. 3(b). There are three signal bands in Fig. 3(b) with central frequencies of $\sim 103, \sim 370$, and $\sim 740 \mathrm{GHz}$ and with full widths at half maximum of $\sim 50$, $\sim 45$, and $\sim 45 \mathrm{GHz}$, respectively. The $\sim 370 \mathrm{GHz}$ frequency corresponds to the acoustic wave vector $q=2 \pi / \Lambda$, where $\Lambda=22.6 \mathrm{~nm}$, assuming the longitudinal acoustic sound velocity along the $c$-axis $\operatorname{In}_{0.12} \mathrm{Ga}_{0.88} \mathrm{~N} / \mathrm{GaN}$ MQWs to be $8360 \mathrm{~m} / \mathrm{s}$, slightly higher than the reported value of $8020-8160 \mathrm{~m} / \mathrm{s}$ for bulk GaN (Refs. 22 and 23) and is in good agreement with a recently reported value in InGaN/GaN MQW. ${ }^{24}$ Furthermore, since the widths of the

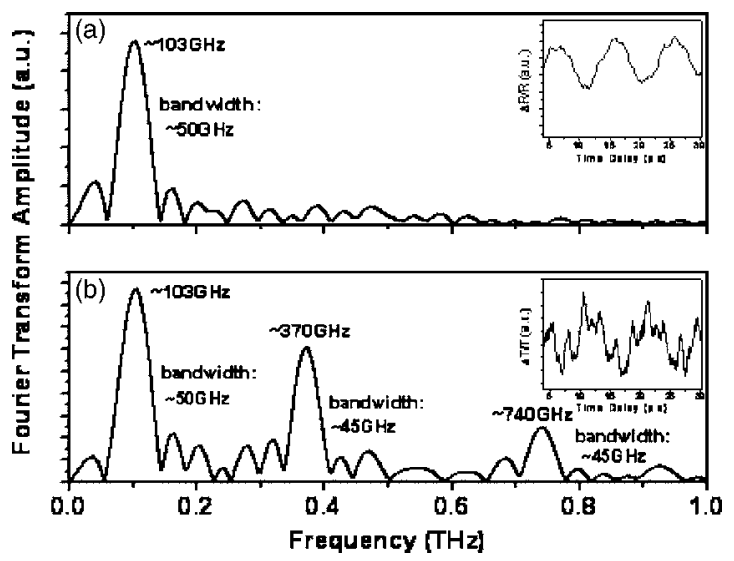

FIG. 3. (a) Fourier transform of the inset of (a), which shows the measured transient reflection change for $\sim 4-30$ ps probe time delay. The absolute magnitude of the relative reflectivity oscillation $\Delta R / R$ is on the order of 3 $\times 10^{-5}$. (b) Fourier transform of the inset of (b), which shows the measured transient transmission change for $\sim 4-30$ ps probe time delay.

to AIP license or copyright; see http://apl.aip.org/apl/copyright.jsp 


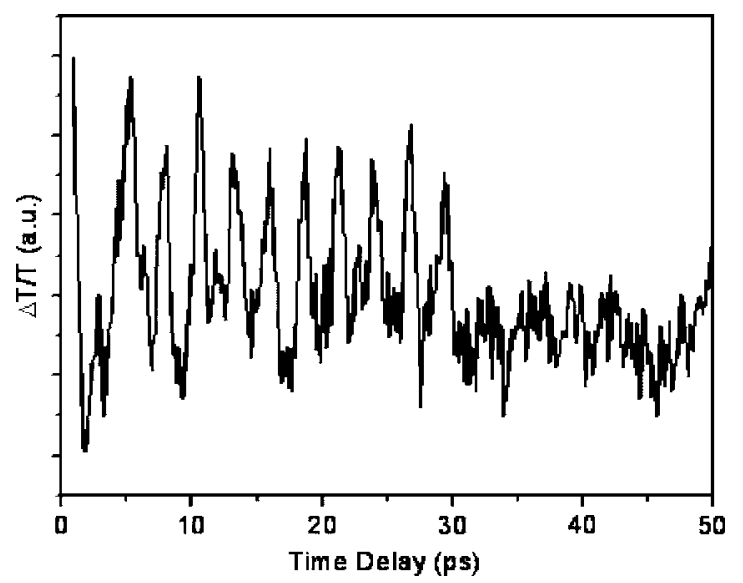

FIG. 4. Measured transient transmission trace with the band at a central frequency of $\sim 103 \mathrm{GHz}$ filtered out. Ten significant peaks, situated between $\sim 4$ and $30 \mathrm{ps}$, correspond to the time when the LAP propagating through the ten equally spaced InGaN quantum wells.

barriers and that of the wells are not the same $(19 / 3.6 \mathrm{~nm})$, in principle, the acoustic spectral sensitivity function of the MQWs has multiple detection bands. With an asymmetric well/barrier ratio, the MQW spatial frequencies are with a fundamental wave vectors of $q=2 \pi / \Lambda$ and its higher harmonics. ${ }^{10}$ With a limited bandwidth phonon source $S(\omega)$, we were able to observe the fundamental frequency of $\sim 370 \mathrm{GHz}$ and its second harmonic, i.e., $\sim 740 \mathrm{GHz}$, being detected by the $\mathrm{In}_{0.12} \mathrm{Ga}_{0.88} \mathrm{~N} / \mathrm{GaN}$ MQW. Moreover, in theory, the bandwidth of these multiple detection bands must be the same, which are determined by the total MQW width $D$. The bandwidths of $\sim 370 \mathrm{GHz}$ band and $\sim 740 \mathrm{GHz}$ band are $\sim 45 \mathrm{GHz}$, which also consists with the expectation that the bandwidth should be equal to $\sim 1.2 v_{a} / D$, where $v_{a}$ is the acoustic velocity in the MQW.

As regards the $\sim 103 \mathrm{GHz}$ signal, we must first understand the detection mechanism of picosecond ultrasonics in transparent (or partially transparent) materials. ${ }^{21,25,26}$ Its detection mechanism is based on the modification of the optical properties in strained materials due to a LAP inside. In previous picosecond ultrasonics studies, the transient reflectivity of the probe beam oscillates when the LAP propagates in the medium, which is the so-called "Brillouin oscillation.," When the optical reflection varies in a transparent system, the optical transmission must change at the same time; therefore, the intensity of the transmitted probe beam also vacillates corresponding to the Brillouin oscillation, and both oscillations have the same frequency. For this reason, we suggest that the $\sim 103 \mathrm{GHz}$ transmission signal comes from the Brillouin oscillation. To verify this, we performed the corresponding transient-reflection two-color pump-probe experiment on the same sample, and the results are shown in Fig. 3(a). There indeed is an $\sim 103 \mathrm{GHz}$ oscillation with an $\sim 50 \mathrm{GHz}$ bandwidth, while the observed Brillouin oscillation is with a $180^{\circ}$ phase shift from that observed in the transmission measurement, supporting our suggestion.

In order to exclusively study the characteristic of the $\mathrm{In}_{0.12} \mathrm{Ga}_{0.88} \mathrm{~N} / \mathrm{GaN}$ MQW acoustic detector in the time domain, we filtered out the $\sim 103 \mathrm{GHz}$ acoustic signals and the result is shown in Fig. 4. There are obviously ten peaks with a period of $\sim 2.67 \mathrm{ps}$, corresponding to the LAP propagating through the ten equally spaced InGaN quantum wells one by one. If there was strong acoustic reflection between $\mathrm{In}_{0.12} \mathrm{Ga}_{0.88} \mathrm{~N}$ and $\mathrm{GaN}$ interfaces, the trace would have more than ten significant peaks. This result suggests that the propagating LAP is insignificantly affected by $\operatorname{In}_{0.12} \mathrm{Ga}_{0.88} \mathrm{~N} / \mathrm{GaN}$ multilayers, and the original profile of the LAP is basically kept during its propagation throught the InGaN MQW.

In summary, we demonstrated that the low-impedancemismatch piezoelectric superlattice can serve as narrow-band detectors for propagating longitudinal CAPs at multiple frequencies corresponding to the fundamental spatial frequency of the superlattice and its higher harmonics, and its detection bandwidth is determined by the total structure width. By optically exciting a broadband propagating longitudinal acoustic pulse from a thin Ni film, we studied the acoustic spectral sensitivity function for optical transmission of a ten-period $\mathrm{In}_{0.12} \mathrm{Ga}_{0.88} \mathrm{~N} / \mathrm{GaN}$ MQW. Because the barriers and wells are of different width, the second detection band, corresponding to the second harmonic of the fundamental frequency, can be resolved.

This project is sponsored by National Science Council of Taiwan under NSC96-2120-M-002-014.

${ }^{1}$ A. Yamamoto, T. Mishina, Y. Masumoto, and M. Nakayama, Phys. Rev. Lett. 73, 740 (1994).

${ }^{2}$ A. Bartels, T. Dekorsy, H. Kurz, and K. Köhler, Phys. Rev. Lett. 82, 1044 (1999).

${ }^{3}$ C.-K. Sun, J.-C. Liang, C. J. Stanton, A. Abare, L. Coldren, and S. P. DenBaars, Appl. Phys. Lett. 75, 1249 (1999).

${ }^{4}$ C.-K. Sun, J.-C. Liang, and X.-Y. Yu, Phys. Rev. Lett. 84, 179 (2000).

${ }^{5}$ O. Matsuda, T. Tachizaki, T. Fukui, J. J. Baumberg, and O. B. Wright, Phys. Rev. B 71, 115330 (2005).

${ }^{6}$ K. Mizoguchi, M. Hase, S. Nakashima, and M. Nakayama, Phys. Rev. B 60, 8262 (1999).

${ }^{7}$ G.-W. Chern, K.-H. Lin, and C.-K. Sun, J. Appl. Phys. 95, 1114 (2004).

${ }^{8}$ C. Thomsen, J. Strait, Z. Vardeny, H. J. Maris, J. Tauc, and J. J. Hauser, Phys. Rev. Lett. 53, 989 (1984).

${ }^{9}$ C. Thomsen, H. T. Grahn, H. J. Maris, and J. Tauc, Phys. Rev. B 34, 4129 (1986).

${ }^{10}$ G.-W. Chern, K.-H. Lin, Y.-K. Hung, and C.-K. Sun, Phys. Rev. B 67, 121303 (2003).

${ }^{11}$ S. Tamura, D. C. Hurley, and J. P. Wolfe, Phys. Rev. B 38, 1427 (1988).

${ }^{12}$ V. Narayanamurti, H. L. Störmer, M. A. Chin, A. C. Gossard, and W. Wiegmann, Phys. Rev. Lett. 43, 2012 (1979).

${ }^{13}$ N. M. Stanton, R. N. Kini, A. J. Kent, M. Henini, and D. Lehmann, Phys. Rev. B 68, 113302 (2003).

${ }^{14}$ N. D. Lanzillotti-Kimura, A. Fainstein, A. Lemaître, and B. Jusserand, Appl. Phys. Lett. 88, 083113 (2006).

${ }^{15}$ S. Mizuno and S. Tamura, Phys. Rev. B 45, 13423 (1992).

${ }^{16}$ A. F. Wright, J. Appl. Phys. 82, 2833 (1997).

${ }^{17}$ V. W. L.Chin, T. L. Tansley, and T. Osatchan, J. Appl. Phys. 75, 7365 (1994).

${ }^{18}$ K.-H. Lin, G.-W. Chern, Y.-K. Huang, and C.-K. Sun, Phys. Rev. B 70, 073307 (2004).

${ }^{19}$ B. A. Auld, Acoustic Fields and Waves in Solids (Wiley, New York, 1973), Vol. 1, p. $358 \mathrm{ff}$.

${ }^{20}$ C.-K. Sun, F. Vallée, L. H. Acioli, E. P. Ippen, and J. G. Fujimoto, Phys. Rev. B 50, 15337 (1994).

${ }^{21}$ H.-N. Lin, R. J. Stoner, H. J. Maris, and J. Tautc, J. Appl. Phys. 69, 3816 (1991).

${ }^{22}$ C. Deger, E. Born, H. Angerer, O. Ambacher, M. Stutzmann, J. Hornsteiner, E. Riha, and G. Fischerauer, Appl. Phys. Lett. 72, 2400 (1998).

${ }^{23}$ Y.-K. Huang, G.-W. Chern, C.-K. Sun, Y. Smorchkova, S. Keller, U. Mishra, and S. P. DenBaars, Appl. Phys. Lett. 79, 3361 (2001).

${ }^{24}$ Ü. Özgür, C.-W. Lee, and O. Everitt, Phys. Status Solidi B 228, 85 (2001).

${ }^{25}$ O. B. Wright, J. Appl. Phys. 71, 1617 (1992).

${ }^{26}$ A. Devos and R. Côte, Phys. Rev. B 70, 125208 (2004). 